East African Medical Journal Vol. 77 No. 11 November 2000

COCKROACH HYPERSENSITIVITY IN ASTHMATICS IN LAGOS, NIGERIA

A. O Adanijo, MBBS, FMCP, Lecturer, Department of Medicine and E.O. Bandele, MBBS, FMCP, FWACP, FCCP, Professor, Consultant Chest

Physician and Head, Respiratory Unit, College of Medicine, Lagos University Teaching Hospital, PMB 12003, Lagos, Nigeria.

Request for reprints to: Dr. A. O. Adanijo, Department of Medicine, College of Medicine, University of Lagos, Lagos, Nigeria.

\title{
COCKROACH HYPERSENSITIVITY IN ASTHMATICS IN LAGOS, NIGERIA
}

\author{
A. O ADANIJO and E.O. BANDELE
}

\begin{abstract}
Background: Cockroach allergy occurs frequently in asthmatics in urban areas, where the level of cockroach infestation is usually high. The prevalence of cockroach allergy, as well as the effect of associated factors on cockroach sensitisation in Nigeria is largely unknown. Objective: To determine the prevalence of cockroach allergy in asthmatics in Lagos.

Design: A prospective case-control study.

Setting: Medical outpatient department of the Lagos University Teaching Hospital, Lagos, Nigeria.

Subjects: Two hundred and two patients with confirmed bronchial asthma and one hundred non-asthmatic control subjects took part in the study.

Intervention: The cockroach allergen was administered on all the subjects using skin prick technique.

Main outcome measures: The provocation of a skin reaction (wcal) fifteen minutes after administering the cockroach antigen.

Results: Amongst the asthmatic patients 90 (44.6\%) had positive skin tests to the cockroach allergen, compared to nine $(9 \%)$ of the control subjects. There was a male preponderance of cockroach sensitivity amongst the asthmatic patients even though the difference between both sexes was not significant. Other highlights of the study included the fact that cockroach sensitivity was related to age, duration of asthma, nocturnal asthmatic attacks, frequency of attacks, and the levels of infestation. There was an insignificant relationship between cockroach sensitivity and the location of residences as well as the income status of the respondents.

Conclusion: There is the need for more studies on cockroach allergy in asthmatics in our environment.
\end{abstract}

\section{INTRODUCTION}

Numerous skin test studies using various allergens have been carried out in the tropics and in developed countries. However, in the tropics, despite the battery of allergens that have been tested, none of the studies has dealt specifically with the cockroach allergen. The emphasis in most of these studies has been on the house dust mite(15). Since cockroaches are common in the tropics(6), and since the cockroach allergen has been found to be an important component of house $\operatorname{dust}(7)$, it becomes imperative to investigate the role of the cockroach in skin sensitivity in this environment. More so since cockroach allergens have been isolated, and their presence in significant quantities in house dust have been demonstrated(7-9). Cockroach hypersensitivity like most other allergic hypersensitivity reactions can be demonstrated by carrying out skin prick tests.

Cockroaches as excitants of allergic reactions now occupy a unique position amongst athropods because of their manifold activities. Cockroaches have been found to provoke clinical reactions as contactant, inhalant, injectant and ingestant allergens. The whole body, faecal extracts, casts and egg shells of the cockroach have been isolated and found to be important sources of allergens in the induction and exacerbation of bronchial asthma(10,11). The cockroach produces antigen specific IgE which not only provokes a hyper sensitivity reaction but can also produce severe allergic bronchial asthma(12). The immunological profile of the cockroach was also elucidated by Helm et al(13) who isolated the cockroach fraction III a heterogeneous protein containing fractions I and II which had been identified by earlier workers(11).

The primary focus of this study was to evaluate the prevalence of cockroach hypersensitivity amongst asthmatics in an urban city like Lagos, whilst looking at the association between this entity and other correlates like age, sex, socio-economic status and the patient's environment to which cockroach allergy has been found to be related.(10,1415). The relationship between the history of exposure to cockroaches and the occurrence of positive skin test results to the cockroach allergen was also evaluated. 


\section{MATERIALS AND METHODS}

This study which was carried out between February 1996 and April 1997, comprised 202 consecutive consenting asthmatic patients between the ages of 15 and 50 years, from the medical out-patient (MOP) clinic of the Lagos University Teaching Hospital (LUTH). The male:female ratio was 1:1.04. They were patients whose history clinical assessment and spirometric studies suggested bronchial asthma. Patients with severe attacks or exacerbations of bronchial asthma and those who had other severe debilitating disease at the time of testing were excluded from the study. So also were patients on steroids, antihistamines and patients with skin diseases and other conditions which might reduce cell mediated immunity.

The control subjects were 100 in number and they were age and sex matched. They comprised normal patients being seen for unrelated and non cardio-respiratory diseases, with no individual or family history of asthma or allergy.

All patients had clinical assessment and spirometric studies done to confirm bronchial asthma. Laboratory parameters like eosinophil count, sputum analysis (where applicable) and chest radiographs were evaluated. A standard questionnaire was administered, which reflected the demographic data, socio economic profile, duration and time of onset of asthma attacks, presence of cockroaches in the homes, severity of infestation, and knowledge of cockroach allergy. The skin test reactions recorded in both the asthmatic patients and control subjects, were grouped under major and minor reactions.

The Epi- Info (version 6) statistical software was used for data entry and analysis. The Chi-square statistics was used to test for associations in the contingency tables and for comparison of proportions. Statistical significance was said to have been achieved when the 'p' value was equal to or less than 0.05 .

The Research and Ethics Committee of the Lagos University Teaching Hospital, Lagos Nigeria, approved the study protocol.

The allergy testing method used for this study was skin prick testing. The Bencard cockroach allergen, serial no J1039 (2702) manufactured by SmithKline Beecham, Brentford England was used. This cockroach antigen was prepared from the periplaneta specie, which is also the specie most commonly seen in the tropics(16). A prick control and histamine solution supplied by the same manufacturer served as negative and positive controls respectively. The modified skin prick technique suggested by Pepys was used(17). An emergency tray was made available in the event of an anaphylactic reaction. In carrying out the test each patient was comfortably seated and the vital signs were ascertained to be normal. The volar aspect of the forearm was used for the test. If there was bleeding the test was repeated at another site. This procedure was carried out for each of the three test solutions using a new lancet for each test. The patient was instructed not to scratch the area. Excess solution was wiped off with sterile cotton wool and the reaction was read off after fifteen minutes with the Bencard $₫$ skin test reaction gauge supplied with the kit.

\section{RESULTS}

Two hundred and two asthmatic patients and one hundred control subjects took part in the study. Amongst the asthmatic patients, there were 104 (51.5\%) females compared to $98(48.5 \%)$ males. The asthmatic cases and the controls were similar in age and sex $(\mathrm{p}=0.38$ and 0.81 respectively).
The case prevalence of cockroach sensitivity was $44.6 \%$ in the asthmatics and $9 \%$ in the control subjects $(\mathrm{p}<0.05)$.

Even though there was no statistically significant difference when the sex distribution of both cockroach sensitive and non-sensitive groups were compared ( $\mathrm{p}=$ $0.07)$ it was observed that more males $(55.6 \%)$ were sensitive to the cockroach allergen than females $(44.4 \%)$. It was also found that majority of the cockroach sensitive asthmatics and the cockroach sensitive controls were in the 41-50 year age group. This was followed by the 21-30 year age group in both populations.

When the influence of socio- economic factors on cockroach sensitivity was evaluated amongst both groups of asthmatics, it was observed that their income and the location of their residences did not influence the positivity of the test $(p=0.46$ and $p=0.84)$. However, most of the cockroach sensitive and non-sensitive asthmatics $(63.3 \%$ and $64.3 \%$ respectively) lived in medium density areas. Majority of the asthmatics in both groups $(71.1 \%$ and $71.4 \%$ ) were also middle income earners.

The time of onset of attacks, the duration of asthma and the increased frequency of attacks were also found to play significant roles in the cockroach sensitive asthmatics. A total of $83.3 \%$ of the cockroach sensitive asthmatics had their attacks occurring more at night compared with $42 \%$ of the non-sensitive asthmatics. (Table 1). The onset of attacks during the day was comparable in both groups of asthmatics ( $5.6 \%$ and 5.4\% respectively). Most of the non sensitive asthmatics $(52.6 \%)$ appeared to have their attacks occurring at variable times (that is day or night or both).

Table 1

Time of onset of attacks in the asthmatic cases

\begin{tabular}{lcc} 
Time of onset & $\begin{array}{c}\text { Cockroach sensitive } \\
\mathrm{n}=90 \\
\%\end{array}$ & $\begin{array}{c}\text { Cockroach non-sensitive } \\
\mathrm{n}=112 \\
\%\end{array}$ \\
\hline Day & $5(5.6)$ & $6(5.4)$ \\
Night & $75(83.3)$ & $47(42.0)$ \\
Variable & $10(11.1)$ & $59(52.6)$ \\
\hline & $90(100)$ & $112(100)$ \\
\hline
\end{tabular}

$\chi 2=39.39 ; \mathrm{df}=2 \mathrm{p} \leq 0.05$

A higher proportion of cockroach sensitive asthmatics (24.4\%), had the longest duration of asthma (>20years) compared with $(4.4 \%)$ of the non- sensitive asthmatics ( $p<0.05)$. Similarly, $24(21.4 \%)$ of the cockroach sensitive asthmatics had the most frequent number of attacks compared with six $(6.7 \%)$ of the non- sensitive asthmatics $(\mathrm{p}=0.01)$.

Table 2 illustrates that dust was observed to provoke more asthmatic attacks in the cockroach sensitive asthmatics than in the non- sensitive group. Majority of the cockroach sensitive asthmatics $55(61.1 \%)$ had their attacks precipitated by dust compared with $44(39.3 \%)$ of their non- sensitive counterparts $(\mathrm{p}<0.05)$. The calculated risk 
of asthmatic attacks being provoked by dust was 1.49 for the cockroach sensitive asthmatics and 0.67 for the cockroach non-sensitive asthmatics. Only five (5.6\%) of the cockroach sensitive cases gave a history of provocation of attacks by cockroaches, none of the cockroach nonsensitive cases gave such history.

Table 2

The relationship between dust provocation of asthmatic attacks and positive skin tests in the asthmatic patients

\begin{tabular}{lcc}
\hline Dust provocation attack & $\begin{array}{c}\text { Cockroach } \\
\text { non-sensitive } \\
\mathrm{n}=112 \\
\%\end{array}$ & $\begin{array}{c}\text { Cockroach } \\
\text { sensitive } \\
\mathrm{n}=90 \\
\%\end{array}$ \\
\hline Yes & $44(39.3)$ & $55(61.1)$ \\
No & $68(60.7)$ & $35(38.9)$ \\
\hline & $112(100)$ & $90(100)$ \\
\hline
\end{tabular}

$\chi 2=9.51 ; \mathrm{p}<0.05$

Table 3 illustrates that the levels of cockroach infestation were significantly higher in the cockroach sensitive subjects (asthmatics and controls). The levels of cockroach infestation of the homes of all the 302 respondents were assessed and grouped into mild, moderate and severe infestation. Majority of the cockroach sensitive and nonsensitive respondents $(52.5 \%$ and $47.3 \%$ respectively) admitted to moderate cockroach infestation of their homes. History was considered positive for significant cockroach exposure if patients reported cockroach infestation of their homes. This was reported by all the $202(100 \%)$ asthmatics and 100 (100\%) controls.

Table 3

Comparison of the levels of cockroach infestation in cockroach sensitive and non sensitive subjects

\begin{tabular}{lcc}
\hline Levels of Infestation & $\begin{array}{c}\text { Cockroach } \\
\text { non sensitive } \\
\text { subjects } \\
\mathrm{n}=203 \\
\%\end{array}$ & $\begin{array}{c}\text { Cockroach sensitive } \\
\text { subjects } \\
\mathrm{n}=99 \\
\%\end{array}$ \\
\hline Mild & $45(22.2)$ & $8(8.1)$ \\
Moderate & $96(47.3)$ & $52(52.5)$ \\
Severe & $62(30.5)$ & $39(39.4)$ \\
\hline & $203(100)$ & $99(100)$ \\
\hline
\end{tabular}

$\chi 2=10.14 ; \mathrm{p}=0.02$

Mild (1- 6 cockroaches apparent); Moderate (7 - 24 cockroaches apparent); Severe ( $>24$ cockroaches apparent)

Of the 302 study population, only four $(1.33 \%)$ had ever heard of cockroach hypersensitivity. They were all control subjects.

\section{DISCUSSION}

Cockroach allergy or hypersensitivity has been found to occur frequently in asthmatics living in urban areas, with prevalence values ranging from $16 \%$ to $55 \%$ ( $16 \%$ in South Africa, $30 \%$ in Mexico, $44 \%$ in New York and $54.9 \%$ in Taiwan)(18-21). It is interesting that such a wide range of prevalence values were obtained in the different locations. Even though this allergen has not been specifically studied in this environment, Warell et al in Zaria, Nigeria in 1979 got significant results when this allergen was tested amongst a battery of other allergens(1). Since cockroaches are ubiquitous in our environment, the levels of exposure might be expected to be high. Significantly high levels of exposure have been reported in some studies in urban centers $(9,22)$. The prevalence of cockroach hypersensitivity obtained in the asthmatic patients in this study was $44.6 \%$. This figure was similar to the $44 \%$ reported in New York(20). However, Warell in Zaria Nigeria got a value of $32 \%$ whilst a much lower value of $16 \%$ was obtained in South Africa $(1,18)$. This may however depend on a number of factors. It is known that intradermal tests give a higher incidence of positive skin test results as opposed to skin prick testing(17). This fact may have a bearing on the eventual prevalence value obtained, because the results will then depend on the type of skin test used in eliciting the hypersensitivity reaction. The value of $44.6 \%$ obtained in this study is therefore thought to be significant since skin prick testing alone was used. A much lower prevalence value of $9 \%$ was obtained in the control subjects. This is comparable to values of $7.5 \%$ and $10 \%$ obtained in other studies $(1,23)$.

Cockroach allergy has been found to be related to a number of factors amongst which are age, sex, and socioeconomic factors $(10,14,15)$. This study showed that more male asthmatics (55.6\%), were sensitive to the cockroach allergen compared with the females $(44.4 \%)$ even though the difference was not significant. A similar pattern was also noted by Kang et al in Chicago(14). There was however a reversal of this gender difference in other studies which showed a female preponderance of cockroach sensitivity $(15,22)$. The reason proffered by those workers was that since cockroaches were found most commonly in the kitchen, and since females spent more time in the kitchen, they were likely to be more exposed and thus more sensitive.

Cockroach hypersensitivity was commoner in the 4150 year age group followed by the 21-30 year age group. A comparable study in Chicago showed the highest prevalence in the 20-35 year old age group(22). However, Kang et al obtained the highest prevalence in a much younger age group (7-12 years)(14). It has been previously noted that the prevalence of cockroach hypersensitivity decreased with age(14), but this study showed an increase with age. Platts Mills et al who showed that there was an increased capacity to elicit skin sensitivity antibodies with increasing age and exposure further corroborates the finding in this $\operatorname{study}(24)$. 
Although, cockroach sensitivity is closely related to a patient's socio economic status $(10,14,15)$, this was not reflected when the location of the residences and the income status of both groups of asthmatics were compared in this study. However, the propensity for cockroach sensitive subjects to live in crowded areas (high and medium density areas) with most of them being middle income earners was noted.

This has been corroborated by other studies $(14,15)$. This distribution may however be a reflection of the distribution of the residences of the study population as a whole.

Nocturnal asthma is a well-recognised entity. There has been a lot of discussion about the cause of the diurnal variation and particularly the nocturnal deterioration in asthma. There have been suggestions that it might be related to feather pillows, mites in bed, circadian corticosteroid variation and also the circadian rhythm of the sympathetic and para- sympathetic nervous system(25). The significant difference in the time of onset of attacks between both groups of asthmatic patients might be an important environmental correlate. Majority of the cockroach sensitive asthmatics $(83.3 \%)$ clearly had their attacks occurring at night compared to $42 \%$ of the nonsensitive group. Even though other factors mentioned earlier contribute to nocturnal attacks of asthma, it should be noted that night - time is the most active time for cockroaches when they shed their skin, cast, pass faeces and lay eggs. Moreover it has already been shown that these body components constitute potent allergens $(10,11)$. Cockroach hypersensitivity might thus have a role to play in nocturnal asthma in this environment.

The duration of asthma is strongly correlated with allergic asthma because a long duration of asthma implies prolonged exposure to allergen. The longer duration of asthma observed in the cockroach sensitive asthmatics in this study might be because of allergen-induced sensitisation at an early age. One of the implicated allergens may be the cockroach allergen. A high frequency of asthmatic attacks in cockroach sensitive asthmatics was also observed in this study. This finding was also reported by Kang et al(22) in Chicago USA. This may imply an increased severity of asthma in them, since the frequency of attacks could also be used as an index of the severity of asthma(25). It is thus possible that cockroach allergy predisposes to severe forms of bronchial asthma.

The finding that dust was the most common provocative factor among asthmatics has been reported in previous studies $(1,2,5,22)$. Table 2 illustrates that more cockroach sensitive asthmatics $(61.1 \%)$ admitted to dust provocation of attacks compared with the non- sensitive asthmatics (39.5\%). Since the cockroach allergen is a well documented component of house dust $(7,9)$, an inference that could be drawn from this is that cockroach allergy may in fact have a role to play in the aetiology of bronchial asthma, since dust samples almost always contain this allergen. However, it would have been desirable to measure the dust levels in the homes of the asthmatics and to actually quantify the levels of cockroach allergen in the dust collected. From the calculated relative risk it can also be deduced that the cockroach sensitive asthmatics were more likely than the non-sensitive group to be subject to dust provocation of attacks

Only $5.6 \%$ of the cockroach sensitive patients specifically mentioned cockroach provocation of asthmatic attacks in different settings. They included three housewives and a middle aged man who noticed attacks whilst cleaning cockroach infested areas of the house like the kitchen and wardrobes.

There was also a young college student who developed an attack whenever she handled cockroaches for her biology practical lesson. It was interesting to note that all these five respondents had major reactions to the cockroach allergen.

Cockroach sensitivity may be a reflection of the variable rates of home infestation and population densities since the frequency of sensitisation appears to be related to the degree of exposure. This frequency of sensitisation also depends on the level of cockroach infestation in the homes. The levels of cockroach infestation of the homes of all the 302 respondents were assessed and grouped into mild, moderate and severe infestation(26). It would have been desirable if the antigen used was prepared from cockroaches obtained from this environment. However, the tests carried out were still valid since inter-specie and intra specie cross-reactivity are well documented in the literature $(8,13,27)$. The fact that all the respondents (including the controls) gave a history of infestation of their homes is not surprising given the poor level of hygiene and the overcrowding in multiple occupation dwellings characteristic of an urban city like Lagos. Even the affluent respondents admitted to the presence of cockroaches (though in lesser quantities) in their homes. No cause and effect relationship was established however because significant exposure can occur without overt evidence of infestation(26). The significant difference in the levels of infestation amongst the cockroach sensitive subjects (cases and controls) and the non-sensitive subjects (cases and controls) illustrated in Table 3 showed that the level of infestation might indeed have a role to play in cockroach hypersensitivity.

The association between cockroach allergy and asthma was demonstrated in this study. It was not surprising that the four respondents who had heard about cockroach allergy or asthma prior to the interview were medical doctors. None of the five respondents who admitted to cockroach provocation of their attacks had heard about this entity. This is not surprising since patients are often unaware of any association between allergen exposure and their symptoms(28).

This study has demonstrated a significant level of cockroach hypersensitivity. There is thus the need for more studies on the cockroach allergen in this environment. Also, it would be necessary to carry out skin tests on asthmatics routinely, using the relevant antigens to the 
patient and his environment, bearing in mind that the predominant offending allergen usually varies with the locality, lifestyle, season and climate. Identification of the implicated allergen or allergens will enable patients recognise the allergen they are sensitive to. They can thus be educated about avoidance measures.

\section{REFERENCES}

1. Warrell D.A., Fawcett I.W., Hamson B.D.W., Agamah A.J., Ibu J.O. and Pope M., et al. Bronchial Asthma in the Nigerian savannah Region. Quart. J Med. 1975; 174:325-47.

2. Coomey J.O.O. and Haddock D.R.W. Skin sensitivity to House dust mite extracts in Ghanaian Asthmatics in Accra. Trans roy. Soc. Trop. Med Hyg. 1973; 67:109-11.

3. Haddock D.R.W. and Onwuka S.I. Skin tests in Nigerian Asthmatics from the equatorial forest zone in Benin Nigeria. Trans roy Soc Trop Med Hyg 1971; 71:32-4.

4. Buchanan D.J. and Jones I.G. Mites and House dust mite allergy in Bronchial Asthma is Northern Zambia. Post Med J. 1974; 50: 680-2.

5. Bandele E.O., Elegbeleye O.O., Williams K.O. and Femi Pearse D. An analysis of Skin Prick Reactions on Asthmatics in Lagos. J. Nat. Med Ass. 1983; 75:511-14.

6. Karl V.F. The Cockroach. In Ten little House mites. 3rd ed London Robert Maxwell 1964. Pp 89-92.

7. Lehrer S.B., Horner E.E., Menon P.K., Oliver J. and Hawk P. Cockroach allergic activity: analysis of commercial cockroach and dust extracts. J. Allergy Clin. Immunol 1991; 88:895-901.

8. Pollart S.M., Platts Mills T.A.E. and Chapman M.D. Identification, quantification and purification of cockroach allergens using monoclonal antibodies. J. Allergy Clin Immunol 1989; 72:83-93.

9. Sanders G., Burge H., Muilenberg M. and Solomon W. Detection of cockroach antigen in commercial house dust extracts by ELISA inhibition. J. Allergy Clin Immunol. 1985; 75:146-9.

10. Menon P., Menon V., Hilman B. and Stankus R. Skin test reactivity to whole body and faecal extracts of American and German cockroaches in atopic asthmatics. Ann Allergy 1991; 67: 573-7.

11. Richman P.G., Khan H.A., Turkeltaub P.C., Malveaureaux F.J. and Baer $\mathrm{H}$. The important source of German Cockroach allergens as determined by RAST analysis. J. Allergy Clin Immunol 1985; 73: $590-5$.
12. Pola J., Valdivieso R. and Zapata C. The specific bronchial challenge in cockroach Asthma. Allergol Immunopathol Madr 1988; 16:171-3.

13. Helm R.M., Bandele E.O., Swanson M.C., Cambell A.R. and Wynn S.R. Identification of a German Cockroach specific Allergen by Human IgE and Rabbit IgE. Int Arch Allerg Appl mmunol 1988; 87: 230-8

14. Kang B.C., Johnson J. and Veres - Thorner C. Atopic profile of inner-city asthma with a comparative analysis of the cockroach sensitive and ragweed sensitive subgroups. J Allergy Clin Immunol 1993; 92:802-11.

15. Garcia D.P., Corbett M.L., Subletti J.L. and Pollart S.J. Cockroach allergy in Kentucky: a comparison of inner-city, suburban and rural small town populations. Ann Allergy 1994; 723:203-8.

16. Vines A.E. and Rees N. Biology of the cockroach. Plant and Animal Biology 4th ed London Pitmans (1978) pp 372-84.

17. Pepys J. Skin testing. Brit. J. Hosp. Med. 1975; 14: 412-7.

18. Fraser B.N. Cockroaches in relation to bronchial asthma in the Durban area. S Afr Med J 1979; 55: 637-8.

19. Williams, G.M, Sierra-Monge J.J. and Navarro B. Skin response to the allergens of the roach in allergic patients. Rev Allerg Misc 1991; 38:139-43.

20. Bernton S.H., McMahon T.F. and Brown H. Cockroach asthma. Brit. J Dis Chest 1972; 66:61-6.

21. Lan J.L., Lee D.T., Wu-ch, Chang C.P. and Yeh C.L. Cockroach hypersensitivity: preliminary study of allergic cockroach asthma in Taiwan. J. Allergy Clin Immunol 1988; 82:736-40.

22. Kang B.C., Wu C.W. and Johnson J. Characteristics and diagnosis of cockroach sensitive bronchial asthma. Ann Allergy 1992; 68: 237-44.

23. Bernton H.S. and Brown H. Insect allergy preliminary studies of the cockroach. J Allergy 1964; 35:506-13.

24. Platts-Mills T.A.E. and Weck A.I. Dust mite allergens and asthma, a worldwide problem. J Allergy Clin Immunol 1989; 83: 416-27.

25. Seaton A., Seaton D. and Leitch A.O. In Crofton and Douglas' Respiratory Disease 4th ed Oxford Blackwell Scientific Publication 1989. Pp 660714.

26. Bernton H.S. and Brown H. Cockroach Allergy II: The relation of infestation to sensitisation S. Med J 1967; 60: 852-7.

27. Lehrer S.B., Horner W.E., Menon P. and Stankus R.P. Comparison of cockroach allergenic activity in whole body and faecal extracts. J Allergy Clin Immunol 1991; 87:574-80.

28. Chapman M.D., Pollart S.M., Lucynska C.M. and Platts-Mills T.A. Hidden allergic factors in the aetiology of asthma. Chest 1988; 94:185-90. 